\title{
Host-agent-vector-environment measures for electronic cigarette research used in NIH grants
}

\author{
Mary L Garcia-Cazarin, ${ }^{1}$ Rachel J Mandal, ${ }^{1}$ Rachel Grana, ${ }^{2}$ Kay L Wanke, ${ }^{1}$ \\ Helen I Meissner ${ }^{1}$
}

'Office of Disease Prevention, National Institutes of Health, Bethesda, Maryland, USA 2Division of Cancer Control and Population Sciences, National Cancer Institute, National Institutes of Health, Bethesda, Maryland, USA

Correspondence to Dr Helen I Meissner, Office of Disease Prevention, National Institutes of Health, 6100 Executive Blvd., Suite 3B01, Bethesda, MD 20892-7530, USA; Hm36d@nih.gov

Received 6 September 2017 Revised 18 November 2017 Accepted 23 November 2017

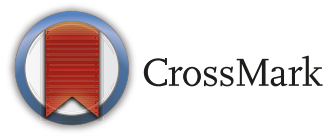

To cite: Garcia-Cazarin ML, Mandal RJ, Grana R, et al. Tob Control Epub ahead of print: [please include Day Month Year]. doi:10.1136/ tobaccocontrol-2017-054032

\section{ABSTRACT}

Objective The purpose of this study is to describe the focus and comprehensiveness of domains measured in e-cigarette research.

Methods A portfolio analysis of National Institutes of Health grants focusing on e-cigarette research and funded between the fiscal years 2007 and 2015 was conducted. Grant proposals were retrieved using a government database and coded using the Host-AgentVector-Environment (HAVE) model as a framework to characterise the measures proposed. Eighty-one projects met the criteria for inclusion in the analysis.

Results The primary HAVE focus most commonly found was Host (73\%), followed by Agent (21\%), Vector (6\%) and Environment (0\%). Intrapersonal measures and use trajectories were the most common measures in studies that include Host measures $(n=59$ and $n=51$, respectively). Product composition was the most common area of measurement in Agent studies $(n=24)$, whereas Marketing $(n=21)$ was the most common $(n=21)$ area of Vector measurement. When Environment measures were examined as secondary measures in studies, they primarily focused on measuring Peer, Occupation and Social Networks $(n=18)$. Although all studies mentioned research on e-cigarettes, most $(n=52 ; 64 \%)$ did not specify the type of e-cigarette device or liquid solution under study.

Conclusions This analysis revealed a heavy focus on Host measures (73\%) and a lack of focus on Environment measures. The predominant focus on Host measures may have the unintended effect of limiting the evidence base for tobacco control and regulatory science. Further, a lack of specificity about the e-cigarette product under study will make comparing results across studies and using the outcomes to inform tobacco policy difficult.

\section{INTRODUCTION}

Decades of research and surveillance on the patterns of tobacco use have made it possible to understand the correlates of smoking and consequent health effects, develop effective interventions for prevention and cessation, and recommend and evaluate tobacco-related programme, policies and legislation. ${ }^{1}$ Much of the data generated by this research provided the evidence needed for passage of the landmark 2009 Family Smoking Prevention and Tobacco Control Act. ${ }^{2}$ The Act gave the U.S. Food and Drug Administration (FDA) immediate authority to regulate cigarettes, cigarette tobacco and roll-your-own and smokeless tobacco and the ability to 'deem' other tobacco products under their jurisdiction.
In August 2016, the FDA extended its regulatory authorities to all other tobacco products, including electronic cigarettes (e-cigarettes), cigars and hookah (waterpipe) and pipe tobacco, and research is underway to help inform regulatory actions regarding these newly deemed products. ${ }^{3}$ A key challenge for regulatory decision-making, however, is to obtain data that not only reliably and accurately assesses the addictiveness, toxicity and appeal of tobacco products but can also be combined to increase statistical power and facilitate the replication and validation of research findings to provide the strongest evidence base possible.

The Tobacco Regulatory Research (TRR) collections of the PhenX Toolkit (http://www.phenxtoolkit.org) described in this journal supplement provide a valuable catalogue of freely available standard measures. When used across different studies, they can facilitate data comparability by ensuring that data are collected in a consistent format. ${ }^{4}$ However, most of the PhenX TRR measures were developed to assess conventional combustible cigarettes because that was the stateof-the-science at the time the toolkit measures were selected. Because research on other tobacco products, such as e-cigarettes, is still in its infancy, it is critical that researchers begin to consider how best to conceptualise and measure factors that could inform regulatory decision-making. As a foundational step in the process of developing consensus measures, characterising the breadth and focus of current research on new tobacco products and the types of measures that investigators are using can be informative. The results can then suggest areas ripe for consensus/standardisation and gaps where new measures are needed.

Measuring the spectrum of factors that influence the use of new tobacco products is necessary to inform tobacco control policy. In the present study, we reviewed research on e-cigarettes using the HostAgent-Vector-Environment (HAVE) organising framework of the PhenX TRR measures to describe the focus and breadth of domains measured in a newly deemed tobacco product. This framework was adopted as an organisational schema by the PhenX Tobacco Regulatory Research Panel, a team of investigators overseeing the PhenX TRR effort. ${ }^{4}$ In this framework, the Host represents the tobacco product user or potential user, ${ }^{5}$ the Agent characterises the tobacco products, ${ }^{6}$ the Vector typifies the tobacco product manufacturers and retailers, ${ }^{7}$ and the Environment refers to macrosocial influences on tobacco uses, such as familial, social, media, legislation and policy influences. ${ }^{8}$ We abstracted 
Web-based NIH database yielded 119 applications containing the following search key words:

electronic cigarettes, electronic nicotine delivery systems, e-cigarettes, and e-cigs combined with "or."

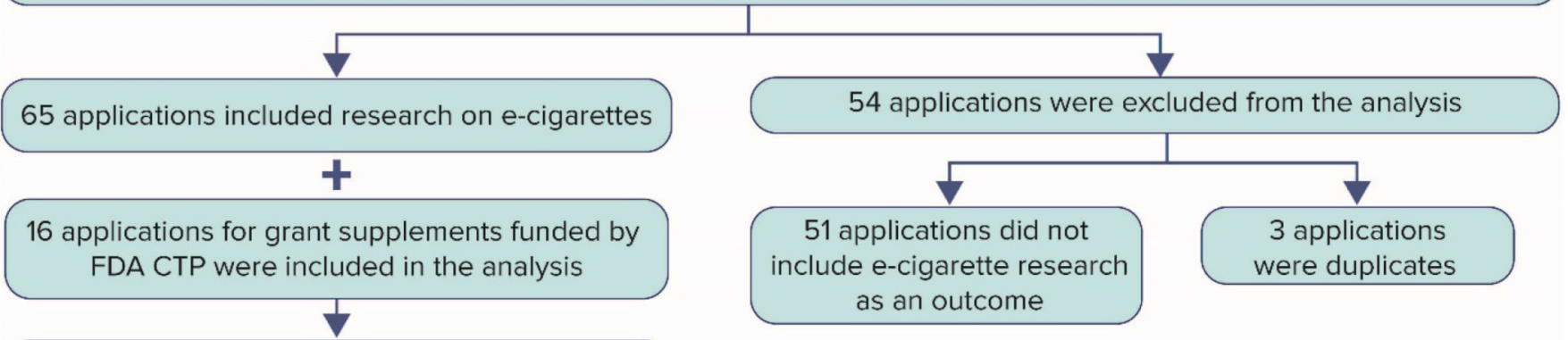

81 applications were considered for the final analysis

Figure 1 Summary of number of e-cigarette grants identified using a National Institutes of Health (NIH) web-based database (FY2007-2015). FDA CTP, Food and Drug Administration's Center for Tobacco Products.

information on measures from funded grant proposals as these documents constitute a useful source of data on the development of this emerging field of inquiry. Grant proposals generally reflect investigators' best intentions to describe the types of measures to be used in developing and evaluating a given study or experiment. Reliance on grant proposals also afforded a more comprehensive look at the research that is underway in this nascent field than would an analysis of the published literature, given the multiple-year lag-time from funding to publication.

\section{METHODS}

This study was undertaken by a team of National Institutes of Health (NIH) staff with expertise in tobacco regulatory science and was supported by the NIH Office of Disease Prevention. We developed a coding instrument using the HAVE model as a framework to characterise the measures proposed in NIH e-cigarette research grants funded between 2007 and 2015. The study was conducted from March 2015 to September 2016. Only funded grant proposals were used in this study based on the premise that they represent the best state-of-the-science. The coding instrument was developed based on FDA-established scientific domains for tobacco regulatory science and HAVE measures adapted from the PhenX TRR collections within the PhenX Toolkit (http://www.phenxtoolkit.org). The initial coding instrument was tested by performing iterative pilot coding of 11 grant proposals and reaching consensus that provided consistency in the interpretation of terms and categories among coders.

Grant proposals were retrieved using a NIH web-based database with detailed information about grant proposals and awards accessible only to staff from NIH and other federal agencies. This database was used to identify grant proposals that included research on e-cigarettes. Search terms included electronic cigarettes, electronic nicotine delivery systems (ENDS), e-cigarettes and e-cigs combined with 'or'. These terms were used to search the title, abstract, specific aims and summary statement from grant proposals funded between fiscal years 2007 and 2015. We included any grant proposal or project that included e-cigarettes regardless of grant mechanism (eg, R01 or K01) or funding source (NIH or FDA). Projects that were part of multicomponent grants (eg, P50) or supplements to a parent grant were also included. For supplements, we only included those funded by the FDA's Center for Tobacco Products (CTP) because there was no systematic way to retrieve administrative supplement information from all NIH Institutes and Centres.

An initial search using the NIH web-based database yielded 119 records containing the selected keywords. Grant proposals were verified by two scientific programme staff as e-cigarette research grants by reading the specific aims and research strategy sections. Fifty-four grant proposals were excluded based on this vetting because the specific aims and research strategy did not include e-cigarettes as one of the products under study or the focus of the grant was not on e-cigarettes even though the keywords could have been mentioned somewhere in the search fields (eg, feedback in summary statement review references a need for the research to consider the advent of e-cigarettes). In addition, three grant proposals were excluded because they were duplicates of ones already included in the database. Sixteen proposals for grant supplements funded by the FDA CTP were also added to the analysis. Such supplements were screened in the same manner as all previous grant proposals by two staff members who read the specific aims and research strategy. In total, 81 grants, projects and supplements were identified for inclusion (figure 1). These 81 records were coded independently by two coders who then met to achieve consensus on all coded variables. Final consensus was entered into an electronic database, and the results were imported into MS Excel for further analysis.

\section{Coding method}

A coding guide and coding sheet were developed by NIH staff and included basic grant information, such as principal investigator name, principal investigator degree and discipline, parent grant mechanism and source of grant funding (NIH or FDA). Grant proposals were also coded to identify their potential to inform policies based on research questions made public in 2012 by the FDA CTP that describe seven CTP Research Priority areas: Diversity of Tobacco Products, Reducing Addiction, Toxicity/Carcinogenicity, Health Consequences, Communications, Marketing, and Economics and Policies (http://www.fda. gov/downloads/tobaccoproducts/newsevents/ucm293998.pdf). The Diversity Priority area was separated into two categories for coding purposes: Chemistry/Engineering and Knowledge, Attitudes, and Behaviors (KAB). The following categories were also coded: project start date, primary population under study 


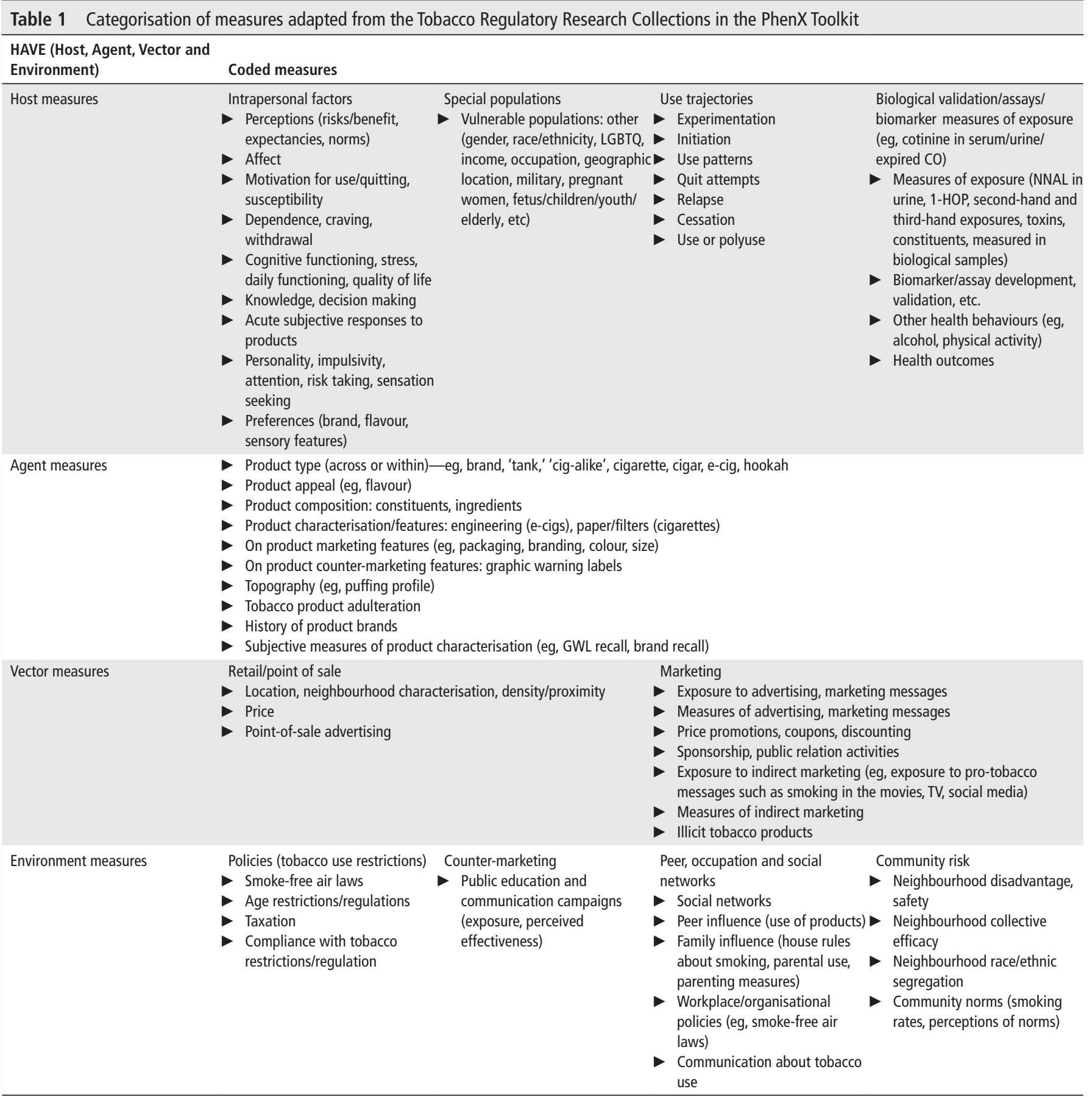

1HOP, 1-hydroxypyrene; GWL, graphic warning labels; NNAL, 4-(methylnitrosamino)-1-(3-pyridyl)-1-butanol.

(coded if the selected population was a focus of the grant or part of the recruitment), type of ENDS and HAVE measures. If a category was not found in the coding sheet, it was specified as the 'other' category.

\section{Coding framework}

Orleans and Slade first described the traditional HAVE epidemiological model as a framework for tobacco prevention and control. ${ }^{9}$ Giovino et al used the HAVE model as a schema for tobacco surveillance, research and evaluation and defined the conceptual framework as an organisational framework for measurement. ${ }^{10}$ This framework was adopted as an organisational schema by the PhenX Tobacco Regulatory Research Panel, a team of investigators overseeing the PhenX TRR effort. ${ }^{4}$ For this portfolio analysis coding, the categorisation of the HAVE measures was adapted from the organisational framework as described by Giovino ${ }^{1}$ and adapted by the TRR Collections in the PhenX Toolkit. Each of these categorisation schemas differs in its assignment of variables that may cross domains (eg, biomarkers of exposure may be considered to fall within both the Host and Agent categories). Table 1 represents all HAVE measures and their categories coded for each grant proposal. Host measures included interpersonal factors, special populations, use trajectories, biological validation, assays and biomarker measures of exposure. Agent measures included product type, appeal, composition, characterisation, features, topography, tobacco product adulteration, history of product brands and subjective measures of product characterisation. Vector measures included 
retail, point of sale and marketing. Environment measures included policies (tobacco use restrictions), counter-marketing, peer, occupation, social networks and community risk.

\section{RESULTS}

Of the 135 grant proposals yielded from the NIH database and FDA CTP-funded grant supplements, 81 met the criteria for inclusion. Table 2 describes the general characteristics of the portfolio. The majority of the studies (75\%) addressing e-cigarettes were funded by the FDA CTP, and most used the R grant mechanism $(58 \%)$ or a project within a $\mathrm{P}$ (centre grant) mechanism (31\%). The principal investigators of these grants came from diverse disciplinary backgrounds. Psychology (clinical and experimental) was the most prevalent background (21\%), followed by medicine (10\%) and public health (10\%). Unsurprisingly, our search did not identify any grant awards prior to 2011. A jump in research on e-cigarettes occurred in 2013, coinciding with a bolus of FDA CTP funding for tobacco regulatory science.

The portfolio addresses research across tobacco regulatory science research domains, and most of the grants (84\%; data not shown) addressed more than one domain. Chemistry/Engineering, KAB, Tobacco Product Marketing and Economics/Policy were never the sole research focus in the portfolio (data not shown). Research on KAB was most prevalent and Economics/ Policy research was least prevalent. Currently, research on e-cigarettes mostly involves human populations, with adults and cigarette smokers mentioned most often. Although all studies in the portfolio mentioned research on e-cigarettes, most $(n=52 ; 64 \%)$ did not specify the type of e-cigarette device or liquid solution under study. Using the HAVE model as the framework, 73\% of the e-cigarette research portfolio was identified as having a primary focus on Host measures. The Environment domain was not a primary focus of any of the studies included in the database. Host measures, whether the primary focus or not, were included in 93\% of all projects, followed by Agent measures (52\%). Vector and Environment measures were included in approximately a third of the portfolio each $(31 \%$ and $28 \%$, respectively).

Figure 2 displays the permutations of Host, Agent and Vector study measures. Of the 59 grants with a Host primary focus, $13(22 \%)$ included no measures on any other HAVE area. Twenty-five $(42 \%)$ of the grants with a Host primary focus also included Agent measures (alone or in combination with Environment and/or Vector measures). When Agent was a primary focus of the research $(n=17)$, the grant most often $(n=14 ; 82 \%)$ included Host measures. Only five grants had a primary focus on Vector, and of these, two included Host and Environment measures, and two included only Host measures.

Figure 3 shows the types of measures proposed in studies by HAVE categories. Because of the small numbers corresponding to specific measures, Host, Vector and Environment measures are collapsed into subcategories (eg, intrapersonal factors vs perceptions or affect) for presentation purposes. Intrapersonal measures and use trajectories were the most common measures in studies that included Host measures $(n=59$ and $n=51$, respectively). Forty-six studies that included Host measures also included biological validation. Product composition was the most common area of measurement in Agent studies $(n=24)$, whereas Marketing was the most common $(n=21)$ area of Vector measurement. When measures of Environment were included, they primarily focused on measuring Peer, Occupation and Social Networks $(n=18)$.
Table 2 Characteristics of e-cigarette portfolio, 2009-2015 ( $\mathrm{n}=81$

\begin{tabular}{|c|c|c|}
\hline Funding source & $\mathrm{n}$ & $\%$ \\
\hline FDA CTP & 61 & 75 \\
\hline NIH & 20 & 25 \\
\hline \multicolumn{3}{|l|}{ Parent grant mechanism } \\
\hline$P(P 50, P 30)$ & 25 & 31 \\
\hline R (R03, R21, R01, R15) & 47 & 58 \\
\hline U (U01) & 2 & 2 \\
\hline K (K01, K07, K99) & 4 & 5 \\
\hline$F(F 31)$ & 3 & 4 \\
\hline \multicolumn{3}{|l|}{ Parent PI terminal degree discipline } \\
\hline Clinical and experimental psychology & 17 & 21 \\
\hline Medicine & 8 & 10 \\
\hline Public health/epidemiology & 8 & 10 \\
\hline Other & 48 & 59 \\
\hline \multicolumn{3}{|l|}{ Project start } \\
\hline 2011 & 1 & 1 \\
\hline 2012 & 8 & 10 \\
\hline 2013 & 28 & 34 \\
\hline 2014 & 24 & 30 \\
\hline 2015 & 20 & 25 \\
\hline \multicolumn{3}{|l|}{ Research domain* } \\
\hline Chemistry and Engineering & 21 & 26 \\
\hline Knowledge, Attitudes, and Behaviors & 42 & 52 \\
\hline Addiction & 17 & 21 \\
\hline Toxicity and Carcinogenicity & 23 & 28 \\
\hline Health Consequences & 20 & 25 \\
\hline Communications & 15 & 18 \\
\hline Tobacco Product Marketing & 17 & 21 \\
\hline Economics and Policy & 7 & 9 \\
\hline \multicolumn{3}{|l|}{ Primary population under study* } \\
\hline Adults (18+) & 46 & 57 \\
\hline Young adults (18-29) & 17 & 21 \\
\hline Adolescents (12-17) & 6 & 7 \\
\hline Dual/polyuser & 10 & 12 \\
\hline ENDS user & 26 & 32 \\
\hline Cigarette smoker & 41 & 51 \\
\hline Vulnerable population (other than age) & 14 & 17 \\
\hline Non-human subject/animal model & 8 & 10 \\
\hline \multicolumn{3}{|l|}{ Other } \\
\hline \multicolumn{3}{|l|}{ Type of ENDS* } \\
\hline Not specified & 52 & 64 \\
\hline Cig-alike and cigarette-shaped & 16 & 20 \\
\hline Tank systems & 9 & 11 \\
\hline Advanced customisable/modifiable tank systems & 4 & 5 \\
\hline Liquid solution for ENDS (e-liquid) & 12 & 15 \\
\hline \multicolumn{3}{|l|}{ HAVE primary focus } \\
\hline Host & 59 & 73 \\
\hline Agent & 17 & 21 \\
\hline Vector & 5 & 6 \\
\hline Environment & 0 & 0 \\
\hline \multicolumn{3}{|l|}{ Includes any HAVE measure } \\
\hline Host & 75 & 93 \\
\hline Agent & 42 & 52 \\
\hline Vector & 25 & 31 \\
\hline Environment & 23 & 28 \\
\hline
\end{tabular}

${ }^{*}$ Multiple options could be selected.

ENDS, electronic nicotine delivery system; FDA CTP, Food and Drug Administration's Center for Tobacco Products; NIH, National Institutes of Health; PI, principal investigator. 


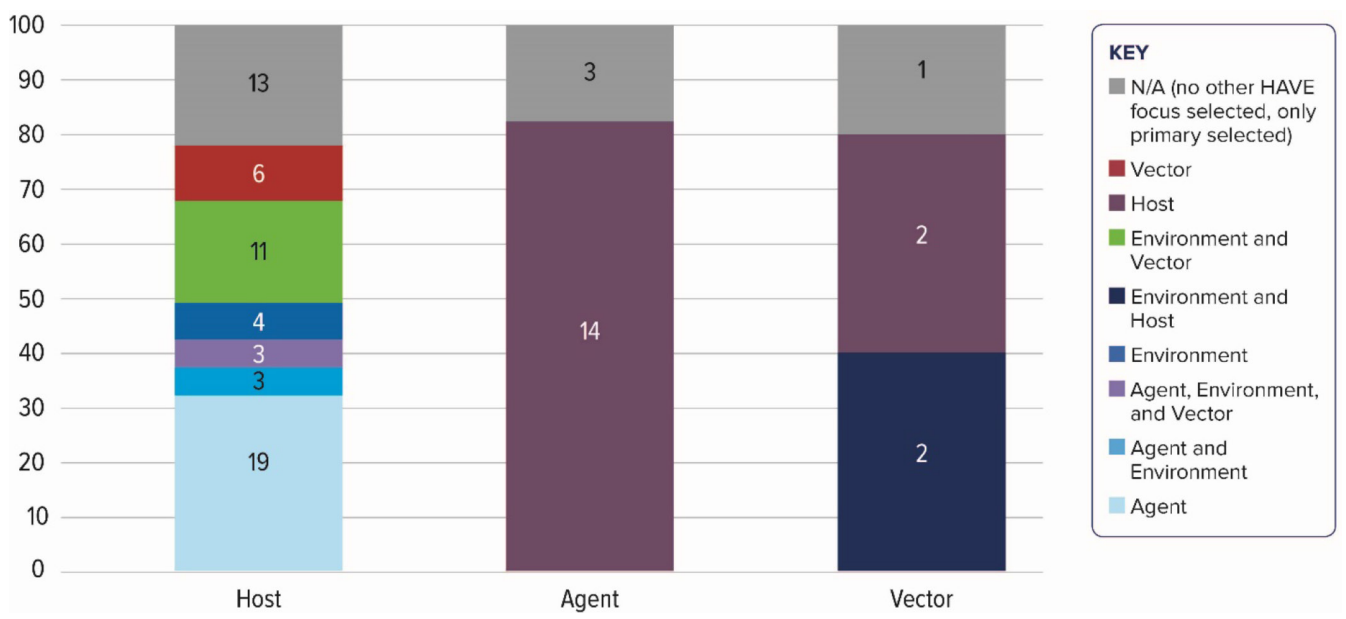

Figure 2 Host-Agent-Vector-Environment (HAVE) measures selected by primary Host-Agent-Vector focus.

Figure 4 displays the HAVE measures by research priority areas. Marketing, Communications, $\mathrm{KAB}$ and Chemistry/ Engineering research included some measures from all HAVE domains, whereas Economics/Policy, Health Consequences and Toxicity included measures from three HAVE areas. Addiction research only included measures from Host and Agent areas (ie, no measures of Environment or Vector were proposed).

\section{CONCLUSIONS}

This paper presents an analysis of the HAVE measures included in NIH grant proposals pertaining to e-cigarettes from 2007 to 2015. Although the grant proposals included measures from all of the HAVE domains, the present analysis revealed a heavy focus on Host measures (73\%) and a lack of focus on measures from the Vector and Environment domains. These findings are consistent with a bibliometric analysis conducted by Cohen et $a l^{11}$ to assess the focus of tobacco research from the 1980 s to the 2000s. During the time period examined, the authors found a decrease in articles focusing on the Agent and an increase in the number of articles focusing on the Host. Overall, however, there were very few articles in either decade focusing on the Environment or the Vector.

Only five grant proposals in our analysis had a primary focus in the Vector domain. This lack of emphasis in the field is consistent with the findings of the workshop presented in Giovino et al. ${ }^{1}$ The workshop participants' highest priority recommendations were in the Vector domain. These included the short-term objective of 'funding research on the measurement and impact of tobacco promotions' and the long-term objective of 'developing a surveillance system that uses validated methods and measures to monitor tobacco industry strategies that promote product use and undermine effects of tobacco control'. ${ }^{1}$ These recommendations were made prior to the introduction of e-cigarettes to the market; however, our analysis shows the need to implement these recommendations remains.

No grant proposals had a primary focus on Environment, and only $28 \%$ of the grant proposals included any Environment measures. FDA CTP funded the majority of grants in this analysis

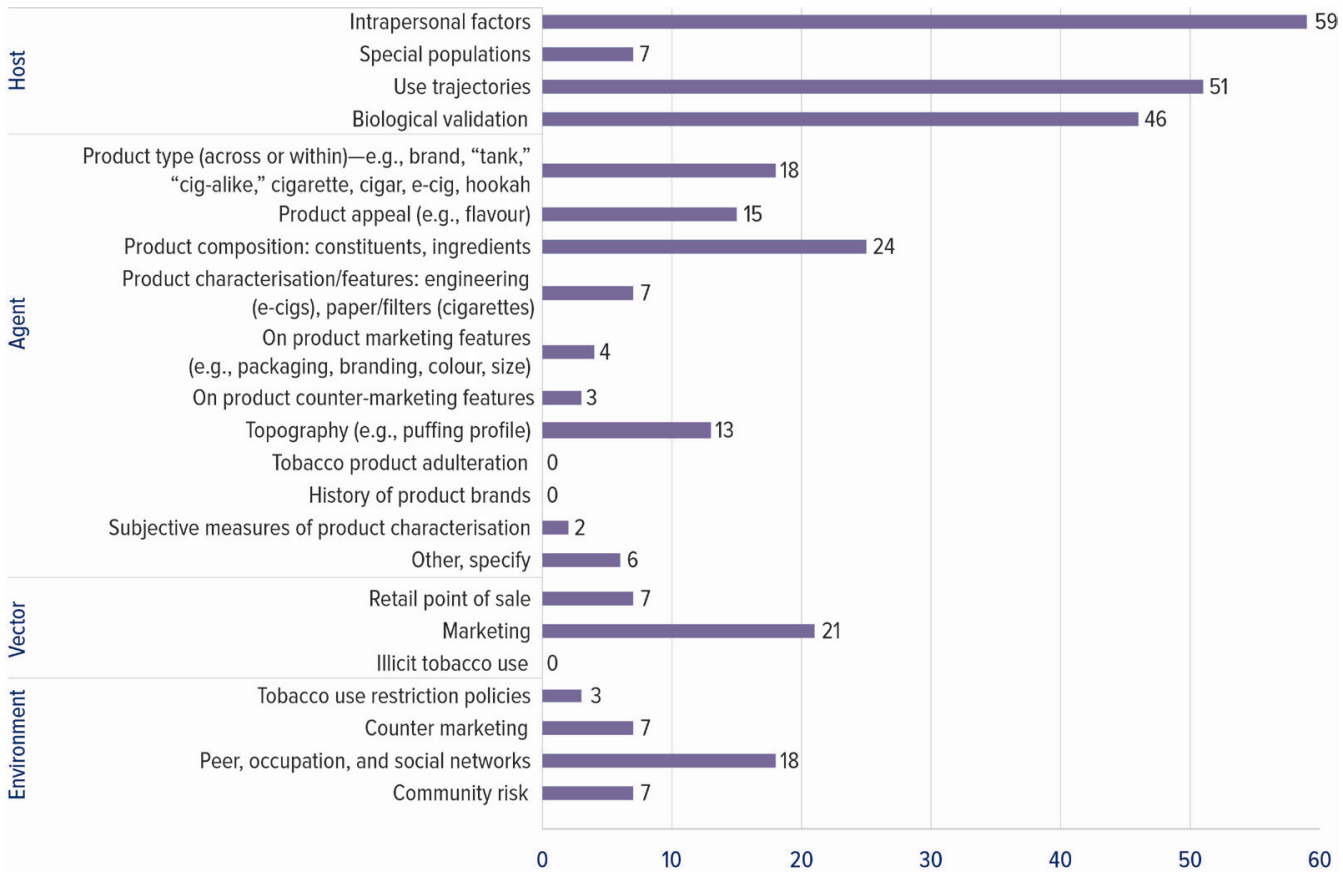

Figure 3 Coded Host-Agent-Vector-Environment measures and subcategories. 


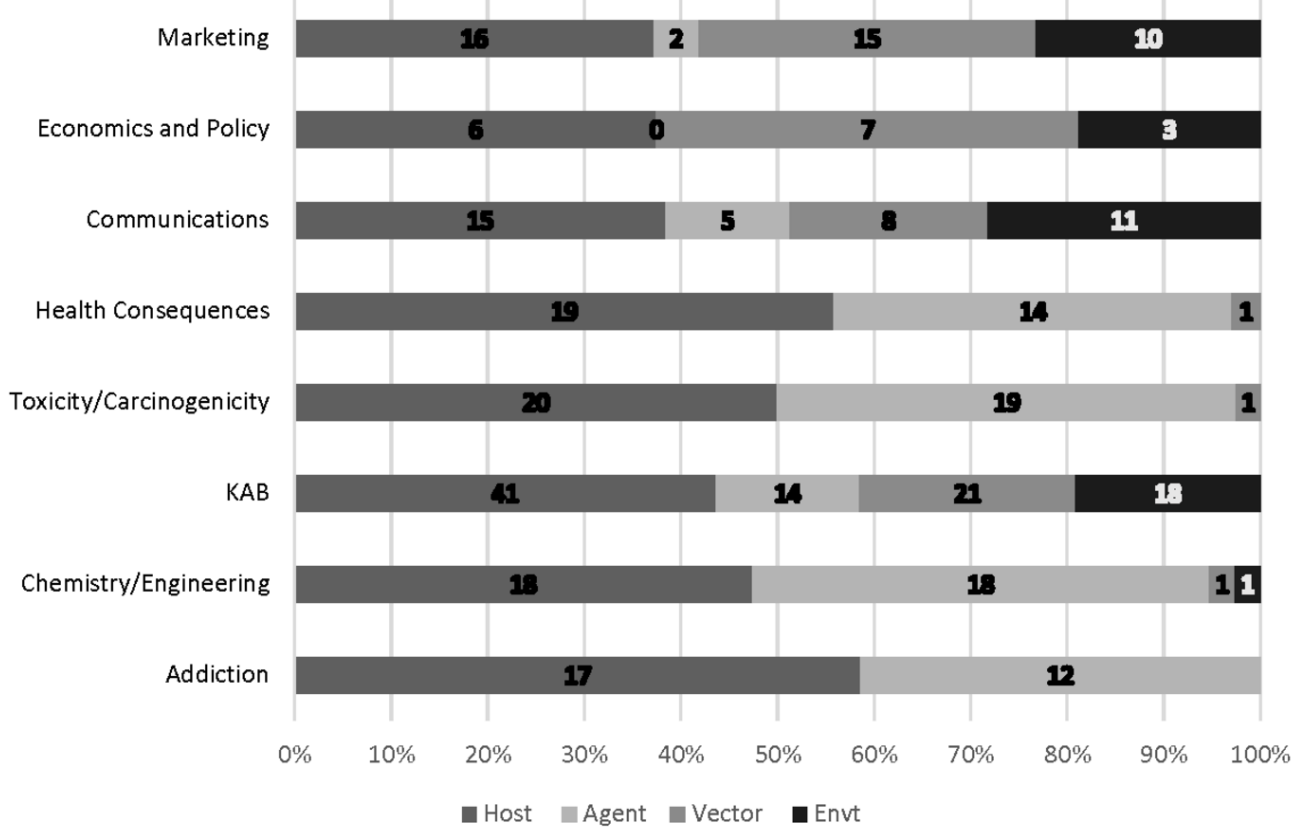

Figure 4 Host-Agent-Vector-Environment measures by research domain. KAB, Knowledge, Attitudes, and Behaviors.

and the Environment domain includes measures outside of their regulatory authority, such as smoke-free air laws and taxation, which may contribute to the lack of studies with a primary focus in the Environment domain. This provides an opportunity for researchers and the NIH to fill this gap in policy research that is applicable to e-cigarettes but outside of the regulatory authority of FDA CTP.

The dearth of measures in the Vector and Environment domains also may reflect the relative infancy of the field of e-cigarette research, with more of the proposed studies focusing on understanding the products and people using them and fewer on the policies or context within which to understand that use or address the issue. The heavy focus on Host measures also may reflect the foundation of expertise on which the research is being built and investigator history in successfully competing for grant funding. It is quite likely that the traditional NIH focus on solicitation of Host-relevant studies and reviewer expectations regarding appropriate research aims contributes to receipt and ultimately funding a greater proportion of grant proposals that measure the Host domain. Still, the predominant focus on measures within the Host domain may have the unintended effect of limiting the evidence base for tobacco regulatory science and tobacco control as a whole. A balanced portfolio of research is needed to provide a comprehensive science base for effective tobacco product regulation ${ }^{12}$ both nationally and internationally. We hope that highlighting the importance of HAVE measures in this nascent field of inquiry will result in more balanced investigation of domains and increase the use of Vector and Environment measures.

Our analysis also revealed a lack of specificity about the e-cigarette product under study, which makes assessing the comprehensiveness of the research portfolio in terms of providing the needed science to establish e-cigarette regulations difficult. Even when a specific product was mentioned, its type and characteristics were not well described, and the authors needed to conduct online searches to determine the product type (eg, cig-alike or tank system). This trend may be problematic for translating the research findings from these studies into regulatory actions. Specificity in terms of the product under study and study conditions is very important for understanding study results and how to use them to provide scientific evidence for regulation.

\section{What this paper adds}

- Effective tobacco control regulation occurs across domains, including policies targeting the Host (tobacco product user or potential user), Agent (tobacco products), Vector (product manufacturers and retailers) and Environment (macrosocial influences on product use). Research measuring the spectrum of factors that influence the use of new tobacco products (eg, e-cigarettes) is necessary to inform tobacco control policy.

- To date, no assessment of the degree to which the four domains are represented within funded National Institutes of Health (NIH) e-cigarette research has been conducted. This study provides the first assessment of Host-AgentVector-Environment measurement domains in funded NIH grants that included research on e-cigarettes.

- Analysis of the portfolio of funded NIH grant proposals on e-cigarette research found a substantial number of studies focused on the Host and Agent domains and a much smaller number of studies focused on the Vector and Environment domains. The analysis also revealed a lack of specificity regarding the e-cigarette product under study.

- More grants focused on the Vector and Environment domains are needed to ensure a balanced portfolio informing tobacco control policy.

- Research proposals focused on all domains should provide specific information to describe the e-cigarette product under study to ensure coherence in the review, and funding decision-making process, and to facilitate the translation and replicability of research findings. 
There were several limitations to our analysis. Although we used popularly accepted terms for e-cigarette products, it is possible that we missed a grant proposal if it used a term for e-cigarettes that was not included among our search terms. However, we are confident that our search captured a majority of funded NIH research on e-cigarettes. We also limited our analysis to proposals of funded NIH grants, which do not have requirements or standards for reporting of planned measures. As such, we noted inconsistencies in the comprehensiveness, detail and specificity in reporting of measures across proposals. Additionally, we limited our analysis to proposals of funded grants, and therefore the results are not reflective of proposals that were not selected for funding. Consequently, the universe of grant proposals in our analysis could reflect a bias of the funding agencies' priorities and not the investigative community. Our scope is limited to research awarded by NIH and therefore represents a slice of the e-cigarette-related research being conducted in the USA and globally. It is possible that NIH grants may not accurately reflect the use of HAVE measures in research funded by other agencies.

Acknowledgements We wish to thank Gary E Swan and Tabitha Hendershot, who provided feedback on our initial coding framework as well as helpful comments on our manuscript. We also gratefully acknowledge the contributions of the RTI editorial team, including Amy Morrow, for providing expert editorial review of the manuscript.

Contributors All authors contributed equally.

Funding The NIH Office of Disease Prevention and the National Cancer Institute provided support for this analysis.

Disclaimer The content is solely the responsibility of the authors and does not necessarily represent the official views of the National Institutes of Health $(\mathrm{NIH})$ or the Food and Drug Administration (FDA).
Competing interests None declared.

Provenance and peer review Not commissioned; externally peer reviewed.

(c) Article author(s) (or their employer(s) unless otherwise stated in the text of the article) 2018. All rights reserved. No commercial use is permitted unless otherwise expressly granted.

\section{REFERENCES}

1 Giovino GA, Biener L, Hartman AM, et al. Monitoring the tobacco use epidemic I. Overview: optimizing measurement to facilitate change. Prev Med 2009;48(1 Suppl):S4-10.

2 US Department of Health and Human Services, Food and Drug Administration. Family smoking prevention and tobacco control act. 2009 http://www.fda.gov/TobaccoPr oducts/Labeling/RulesRegulationsGuidance/ucm246129. htm (accessed Feb 2017).

3 Food and Drug Administration, HHS. Deeming tobacco products to be subject to the Federal Food, Drug, and Cosmetic Act, as amended by the Family Smoking Prevention and Tobacco Control Act; restrictions on the sale and distribution of tobacco products and required warning statements for tobacco products. Final rule. Fed Regist 2016;81:28973-9106.

4 Hamilton CM, Strader LC, Pratt JG, et al. The PhenX Toolkit: get the most from your measures. Am J Epidemiol 2011;174:253-60.

5 Delnevo CD, Bauer UE. Monitoring the tobacco use epidemic III: the host: data sources and methodological challenges. Prev Med 2009;48(1 Suppl):S16-23.

6 Stellman SD, Djordjevic MV. Monitoring the tobacco use epidemic II: the agent: current and emerging tobacco products. Prev Med 2009;48(1 Suppl):S11-15.

7 Cruz TB. Monitoring the tobacco use epidemic IV. The vector: tobacco industry data sources and recommendations for research and evaluation. Prev Med 2009;48(1 Suppl):S24-34.

8 Farrelly MC. Monitoring the tobacco use epidemic V: the environment: factors that influence tobacco use. Prev Med 2009;48(1 Suppl):S35-43.

9 Oxford University Press. In: Orleans CT, Slade J, eds. Nicotine addiction: principles and management. New York: Oxford University Press, 1993. Preface, page ix.

10 Giovino GA. Epidemiology of tobacco use in the United States. Oncogene 2002;21:7326-40.

11 Cohen JE, Chaiton MO, Planinac LC. Taking stock a bibliometric analysis of the focus of tobacco research from the 1980s to the 2000s. Am J Prev Med 2010;39:352-6.

12 Berman ML, Kim AE. Bridging the gap between science and law: the example of tobacco regulatory science. J Law Med Ethics 2015;43(Suppl 1):95-8. 